\title{
Paper
}

\section{Estimating Behavior Considering Multiple Possession Positions of smartphones Using Machine Learning}

\author{
Rui Kitahara $^{* \dagger}$ Member, $\quad$ Seilchi SerikawA ${ }^{\dagger}$ Member \\ LIFENG ZHANG ${ }^{\dagger}$ Member
}

(Received December 15, 2021, revised January 21, 2022)

\begin{abstract}
In recent years, many people have owned wearable devices such as smartphones and smartwatches, in which the built-in sensors were installed. Moreover, such kind of build sensors has been used for various researches like height estimation, road condition estimation, and so on. In addition, it also conducted a behavioral estimation of smartphone-owners, possession location estimation, and person estimation. However, in the case of behavioral estimation, most of them use data measured by fixing the possession position at a single location, and behavioral estimation that takes the possession position of the terminal into account has not been conducted. It is thought that users possess terminals such as smartphones in multiple locations. Therefore, the purpose of this study is to perform a behavioral estimation that considers the multiple possession locations of a user's smartphone. Also, suppose it is possible to estimate a user's behavior by considering the multiple possession locations of the smartphones. In that case, it may be possible to change notifications such as emails and phone calls on smartphones according to the user's state. As a result of classifying 12 classes using LSTM[1], a combination of the GAF[2] algorithm and ResNet50[3], and a combination of the GAF algorithm and Mdk-ResNet[4], the combination of the GAF algorithm and Mdk-ResNet had the highest accuracy, with Accuracy: $98.892 \%$.
\end{abstract}

Keywords: Human Activity Recognition,Machine Learning

\section{Introduction}

In recent years, many people have come to own smartphones and wearable devices such as smartwatches. According to a survey by NTT DoCoMo[5], the percentage of smartphones among cell phones owned was only $4.4 \%$ as of February 2010, but as of January 2021, it was $92.8 \%$. According to the Cabinet Office's Consumer Trend Survey for FY2021[6], the smartphones ownership rate is $75.6 \%$ for single-person households and $88.9 \%$ for families with two or more members. By age group of the head of household, even the group with the lowest smartphones ownership rate, "single-person household and 70 years old or older," has $53.5 \%$, more than half of which own a smartphone, clearly indicating that smartphones are becoming more popular. When it comes to smartwatches, according to a survey by MMD Research Institute[7], 38.0\% of men and 32.6\% of women own a smartwatch by gender. In addition, sales of smartwatches will exceed 2 million units by FY2020, compared to less than 1 million units in FY2015. Furthermore, it is expected to reach 3.025 million units in FY2021 and 4.9 million units in FY2025[8]. Although not as widespread as smartphones, smartwatches are also owned by many people and will continue to increase. With the spread of such terminals, various efforts have been made to use the built-in

\footnotetext{
*Corresponding: kitahara.rui166@mail.kyutech.jp

$\dagger$ Kyushu Institute of Technology

(zhang@elcs.kyutech.jp)
}

sensors. For example, efforts are being made to estimate a person's behavior, state, and height and estimate snow cover on roads. Some systems use sensors to help watch over the elderly. One of the advantages of using sensors is that privacy is protected compared to data acquisition by cameras. Next, we describe below some previous studies on person behavior estimation and individual estimation that are highly relevant to this research.

\subsection{Related Study}

\subsubsection{A Study of Identification of Pedestrian by Using} 3-axis Accelerometer[9]

Iwamoto et al. have studied features for estimating the possession position of pedestrians-owned smartphones using a build-in 3-axis acceleration sensor and have evaluated actual pedestrian estimation using these features. In estimating the possession position of a smartphone, the maximum and minimum values of the 3 -axis acceleration sensor, the average value in each of the three axes, and the amount of change are treated as features. There are six different possession positions set up. J4.8, SMO and Naïve were used as classifiers, and the accuracy was $94.4 \%, 96.6 \%$, and $94.4 \%$. The highest accuracy of $97.7 \%$ was obtained for user estimation by using J4.8, SMO, and Naïve classifiers together with FFT.

1.1.2 Human Activity Recognition Based on Gramian Angular Field and Deep Convolutional Neural Network $\mathrm{H}$. Xu et al. have proposed a new network called Mdk- 
Table 1: Comparison with previous studies

\begin{tabular}{cccc}
\hline & Iwamoto & H. Xu & This Study \\
\hline Possession Positions Estimation & $\bigcirc$ & $\times$ & $\bigcirc$ \\
Behavior Estimation & $\times$ & $\bigcirc$ & $\bigcirc$ \\
Person Estimation & $\bigcirc$ & $\times$ & $\times$ \\
\hline
\end{tabular}

ResNet for the WISDM dataset[10], which is a large-scale behavior classification dataset created by J.RKwapisz et al. The WISDM dataset consists of 29 subjects performing 6 classes of behaviors: walking, jogging, stair climbing, stair descending, sitting, and standing. Using Mdk-ResNet, the results were $96.83 \%$, which is $9.8 \%$ higher than the previous multilayer perceptron classification results.

\subsection{Purpose of this study}

Although behavioral estimation has been conducted in various previous studies, all of them limit the possession location of the acquisition device to a single location. Table 1 shows a comparison between previous studies and this study. However, in reality, users carry their smartphones in various locations. In this research, we aim to estimate the user's behavior by considering the position of the smartphones. Suppose the behavior estimation can be performed even when putting the smartphone in various possession positions. It will be possible to switch the phone reaction according to the user's situation, such as phone calls and email notifications.

\section{Pricinple}

\subsection{GAF Algorithm}

Z. Wang proposes the GAF algorithm to convert 1D timeseries data into $2 \mathrm{D}$ image data.

First, given a time series data consisting of $\mathrm{n}$ real-valued observation $\operatorname{data}\left(X=x_{1}, x_{2}, \ldots, x_{n}\right)$, normalize the data so that all the values fall in the interval $[-1,1]$ or $[0,1]$.

$$
\begin{gathered}
\tilde{x}_{-1}^{i}=\frac{x_{i}-\max (X)+\left(x_{i}-\min (X)\right)}{\max (X)-\min (X)} \\
\text { or } \quad \tilde{x}_{0}^{i}=\frac{x_{i}-\min (X)}{\max (X)-\min (X)}
\end{gathered}
$$

Next, the normalized 1D time-series data is transformed from a Cartesian Coordinate system to a Polar Coordinate system.

$$
\left\{\begin{array}{cc}
\phi=\arccos \left(\tilde{x}_{i}\right),-1 \leq \tilde{x}_{i} \leq 1, & \tilde{x}_{i} \in \tilde{X} \\
r=\frac{t_{i}}{N}, & t_{i} \in \mathbb{N}
\end{array}\right.
$$

Here, when transformed to the Polar Coordinate system, the value of $\phi$ is $[0, \pi / 2]$ when normalized to $[0,1]$, and the value of $\phi$ is $[0, \pi]$ when normalized to [-1,1]. After transforming the normalized time series into a Polar Coordinate system, each temporal correlation can be identified in terms of angle by calculating the sum and difference of the trigonometric functions between each sampling point. Based on the above, Gramian Summation Angular Field (GASF) and

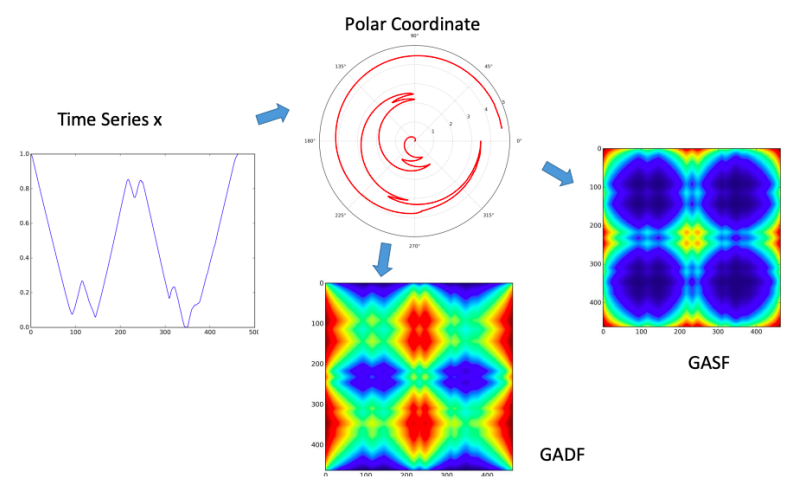

Figure 1: An example using the GAF algorithm

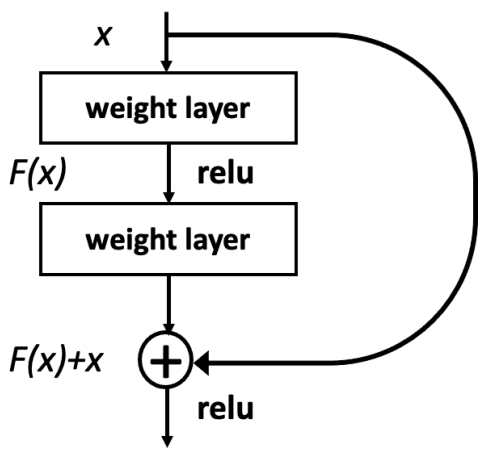

Figure 2: Shortcut Connection

Gramian Difference Angular Field (GADF) are defined.

$$
\begin{aligned}
\mathrm{GASF} & =\left[\cos \left(\phi_{i}+\phi_{j}\right)\right] \\
& =\tilde{X}^{\prime} \cdot \tilde{X}-\sqrt{I-\tilde{X}^{2^{\prime}}} \cdot \sqrt{I-\tilde{X}^{2}} \\
\mathrm{GADF} & =\left[\sin \left(\phi_{i}-\phi_{j}\right)\right] \\
& =\sqrt{I-\tilde{X}^{2}} \cdot \tilde{X}-\tilde{X}^{\prime} \cdot \sqrt{I-\tilde{X}^{2}}
\end{aligned}
$$

Figure 1 shows an example of an output image (GASF • GADF) from 1D time-series data, converted to Polar Coordinates and transformed by the GAF algorithm.

\subsection{ResNet}

The Residual Network (ResNet) introduced a Shortcut Connection mechanism to solve the vanishing gradient by directly adding the input of the front layer to the back layer. Figure 2 shows the rough structure of Shortcut Connection. It was invented by $\mathrm{H}$. Kamining and was the winning model in ImageNet of ILSVRC in 2015. ResNet also consists of repeating residual blocks, and the residual blocks are a combination of convolutional layer and Skip Connection. It consists of two branches and adds up the elements of each. 


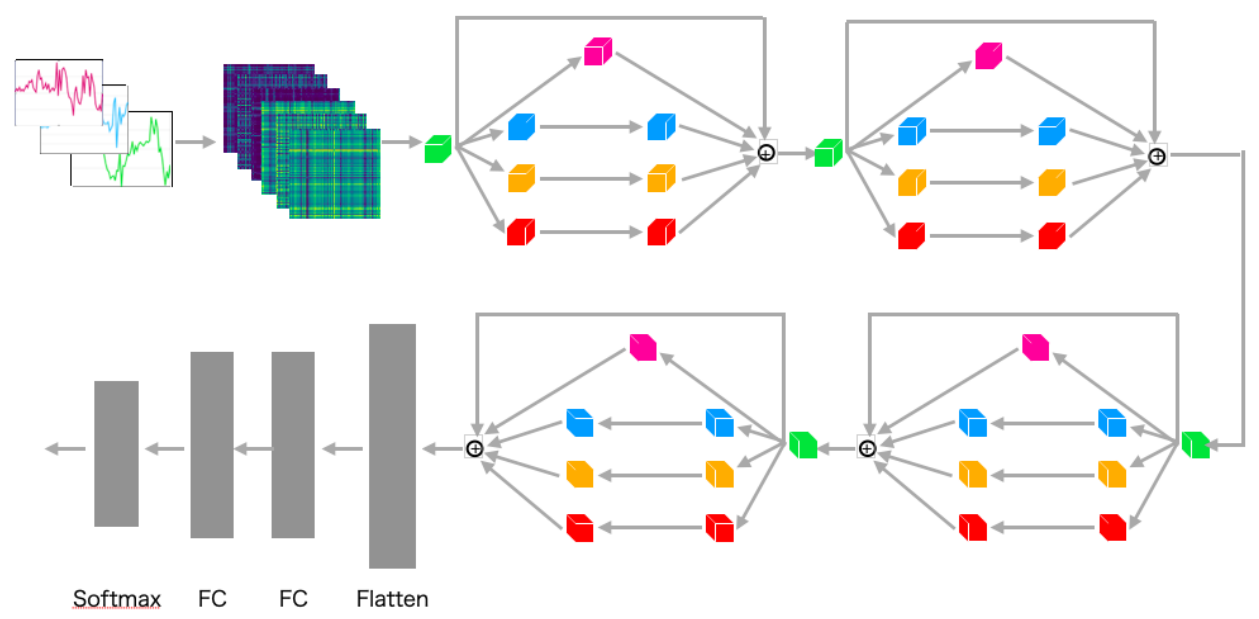

Figure 3: Mdk-ResNet

One of the residual blocks combines convolutional layers and the other is an Identity function. In this way, even if the additional layers do not need to be transformed, we can just set the weights to zero. By introducing the residual blocks, we can push the depth limit of the layers and thus improve the accuracy. As a result, the problem of gradient vanishing, which was caused by deepening the network, has been solved, and even deep networks can now be trained. In addition, many recently proposed networks, such as DenseNet, which is an improved version of ResNet, are based on ResNet and have significantly contributed to the construction of networks with deep layers.

\subsection{Mdk-ResNet}

Mdk-ResNet is a new network proposed by $\mathrm{H}$. Xu et al. with the network structure as shown in Fig. 3. It is used to classify human behavior based on large datasets by classifying $2 \mathrm{D}$ images transformed by the GAF algorithm from 1D time-series data. By combining the GAF algorithm with Mdk-ResNet, we improved the performance on timeseries datasets such as WISDM, UCI HAR, and OPPOTUNITY. Compared to traditional machine learning networks, the combination of the GAF algorithm and Mdk-ResNet has been shown to improve classification accuracy because it is able to extract features between different sampling points.

\subsubsection{Dilated Convolution[11]}

Increased network parameters and computational complexity arise when using large convolutional kernels. There is also the information loss problem that usually occurs when using a pooling layer. F. Yu et al. proposed the dilated convolution that expands the receptive field without loss of information. The schematic diagram of dilated convolution is shown in Fig. 4 Dilated Conv solves the aforementioned problem by allowing the convolutional output to contain an even wider range of information by varying the kernel spacing.

\subsubsection{Mdk-Res Module}

The Mdk-module is inspired by the Inception module of

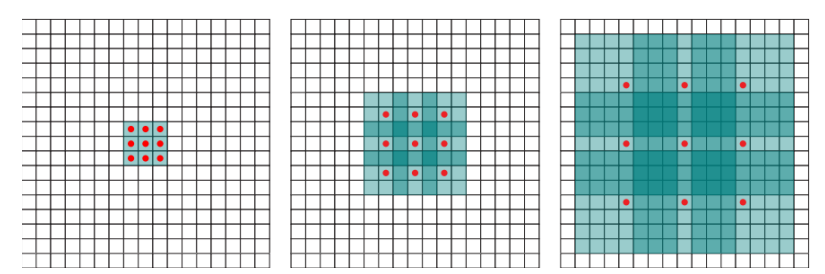

Figure 4: Dilated Convolution

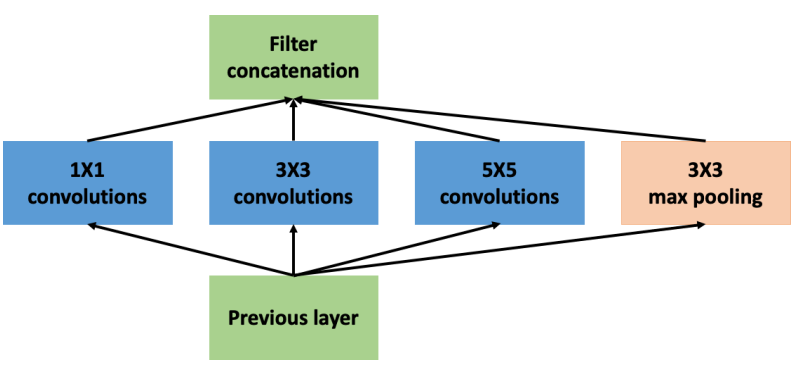

Figure 5: Inception module of GoogleNet

GoogleNet[12]. As shown in Fig. 5, the Inception module performs multiple operations with multiple filter sizes. This improves on the shortcomings of conventional convolutional neural networks where the image size decreases as the convolutional layers get deeper. In the Mdk-Res module, the features of the previous layer are input and processed by four convolutions as shown in Fig. 6 The results of the four blocks and the input features are then used as the output. Set the hyperparameter padding to "same" and stride to " 1 " for the convolution of the four blocks so that the output dimensions are the same. Padding is the process of enclosing pixels around the pixels of the input image, making the size of the image invariant and allowing pixels at the edges of the image to be convoluted as well. If padding is "same", it means that the output will be the same length as the original input. Stride is the interval at which convolution is applied, and stride is " 1 " is to move the filter by one interval. If we put the height and width of the output 


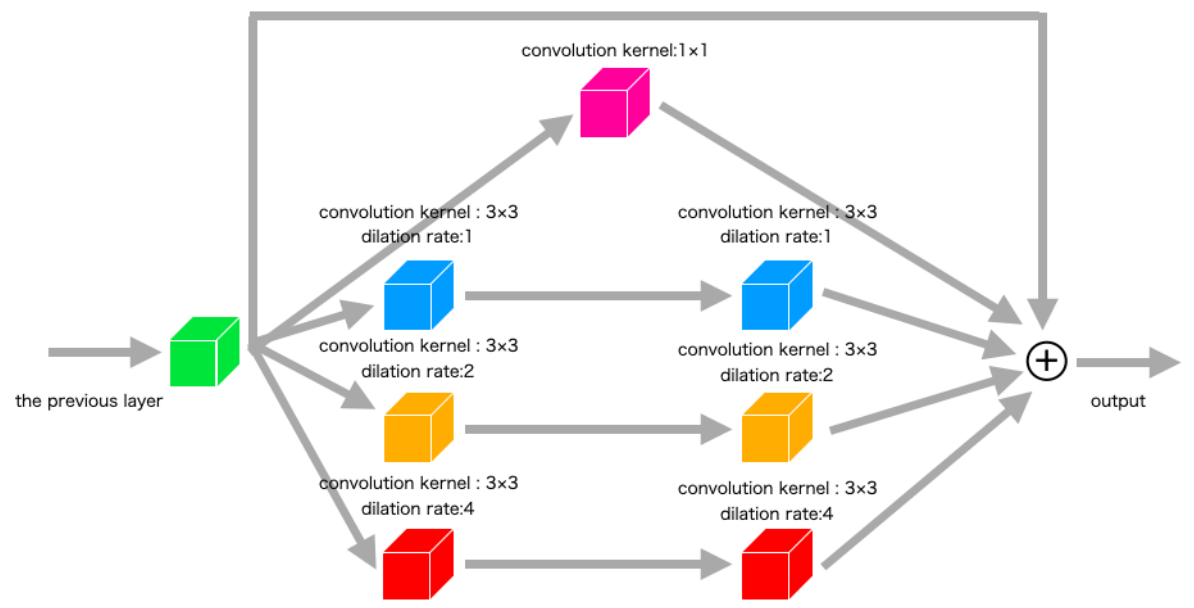

Figure 6: Mdk-Module

size as $Q_{h}, Q_{w}$, the height and width of the filter size as $F_{h}$, $F_{w}$, the padding as $P$, and the stride as $S$, we can calculate as follows.

$$
\begin{aligned}
& Q_{h}=\frac{H+2 P-F_{h}}{S}+1 \\
& Q_{w}=\frac{W+2 P-F_{W}}{S}+1
\end{aligned}
$$

The green block is the previous layer of the network, the peach block has a kernel size of $1 \times 1$, the blue block has a kernel size of $3 \times 3$, the yellow block has a kernel size of $3 \times 3$, dilation rate is 2 , and the red block has a kernel size of $3 \times 3$, dilation rate The Mdk-Res module uses multiple normal and expanded convolution kernels simultaneously. As a result, it helps to improve the capability of the network to extract features at various scales. In addition, as with regular ResNet, residual learning is used to suppress the gradient loss problem.

\subsection{LSTM}

Every RNN is a repeating chain of neural network modules. In a standard RNN, this repeating module has a straightforward structure, a single tanh layer. LSTMs also have a chain-like structure, but the repeating modules have a different structure. Specifically, the LSTM iterative module contains four interacting layers: Memory Cells, Input Gates, Output Gates, and Forgetting Gates. It has the good feature of being able to learn long-term dependencies. Therefore, in many cases, it gives better results than the standard RNN. As a result, it has been used for stock price prediction.

\section{Experiment}

\subsection{Data Acquisition}

In this experiment, we set four locations where users are likely to carry their smartphones. The four locations are: "The Front Pocket of Your Pants (off Screen)," "The Back Pocket of Your Pants (off Screen)," "Holding it in Your Hand and Waving Your Arm (on Screen)," and "Holding it in Your Hand and Looking at the Screen". And, we selected three types of behaviors that are considered to be commonly performed in daily life. There are three types of behaviors: "Walking," "Stair Climbing," and "Stair Descending". A total of 12 classes were set up by combining each of the four types of smartphones possession positions and three types of behavior. The 3 -axis acceleration in these settings was measured with an iPhone SE (second generation). The sampling rate was set to $20 \mathrm{~Hz}$, referring to the WISDM data set of previous studies. For five subjects, approximately 60 seconds of data were acquired per class. From the acquired data, we set the window size to 64 and the overlap to $50 \%$, referring to the study by $\mathrm{H}$. $\mathrm{Xu}$ et al., and finally generated 9468 data in total for 12 classes. The LSTM was trained using three multivariate variables: acceleration $\mathrm{x}$, acceleration $\mathrm{y}$, and acceleration $\mathrm{z}$. The acquired acceleration data were converted from time-series data to $2 \mathrm{D}$ images using the GAF algorithm and trained with ResNet50 and Mdk-ResNet. Since GADF and GASF images were generated from acceleration $\mathrm{x}$, acceleration $\mathrm{y}$, and acceleration z, six images were generated per time series. For LSTM, ResNet50, and Mdk-ResNet, the ratio of data for training, validation, and testing was set to $6: 2: 2$. Adam was used as the optimization function, and the learning rate was set to 0.001. To prevent overtraining, Early Stopping was used.

\subsection{Evaluation Index}

This experiment used four evaluation indices (Accuracy, Macro-Recall, Macro-Precision, and Macro-F1). The calculation method of the evaluation indices is shown below. In the formulas, $T P$ is True Positive (when the model predicts Positive and the correct answer is Positive), $T N$ is True Negative (when the model predicts Negative and the correct answer is Negative), FP is False Positive (when the model predicts Positive and the correct answer is Negative) and $F N$ is False Negative (when the model predicts Negative and the correct answer is Positive).

$$
\begin{aligned}
\text { Accuracy } & =\frac{T P+T N}{T P+T N+F P+F N} \\
& =\frac{T P+T N}{N}
\end{aligned}
$$


Table 2: Explanation of the Confusion Matrix legend

\begin{tabular}{lll}
\hline & Behavior & Locations \\
\hline A & Stair Descending & The Back Pocket of Your Pants \\
B & Stair Climbing & The Back Pocket of Your Pants \\
C & Walking & The Back Pocket of Your Pants \\
D & Stair Descending & The Front Pocket of Your Pants \\
E & Stair Climbing & The Front Pocket of Your Pants \\
F & Walking & The Front Pocket of Your Pants \\
G & Stair Descending & Holding Smartphones in Your Hand and Looking at the Screen \\
H & Stair Climbing & Holding Smartphones in Your Hand and Looking at the Screen \\
I & Walking & Holding Smartphones in Your Hand and Looking at the Screen \\
J & Stair Descending & Holding smartphones in Your Hand and Waving \\
K & Stair Climbing & Holding smartphones in Your Hand and Waving \\
L & Walking & Holding smartphones in Your Hand and Waving \\
\hline
\end{tabular}

Table 3: Evaluating the trained model with test data

\begin{tabular}{cccc}
\hline & LSTM & Mdk-ResNet & ResNet50 \\
\hline Accuracy(\%) & 98.417 & 98.892 & 95.620 \\
Macro-Recall(\%) & 98.426 & 98.482 & 94.695 \\
Macro-Precision(\%) & 98.417 & 98.498 & 94.499 \\
Macro-F1(\%) & 98.416 & 98.458 & 94.558 \\
\hline
\end{tabular}

$$
\begin{gathered}
\text { Macro_Recall }=\frac{1}{N} \sum_{i=1}^{N} \text { recall }_{i} \\
=\frac{1}{N} \sum_{i=1}^{N} \frac{T P}{T P+F N} \\
\text { Macro_Precision }=\frac{1}{N} \sum_{i=1}^{N} \text { precision }_{i} \\
=\frac{1}{N} \sum_{i=1}^{N} \frac{T P}{T P+F P} \\
\text { Macro_F1 }=\frac{1}{N} \sum_{i=1}^{N} F 1_{i} \\
=\frac{1}{N} \sum_{i=1}^{N} \frac{2 \times \text { recall } \times \text { precision }}{\text { recall }+ \text { precision }}
\end{gathered}
$$

\subsection{Resluts}

The experimental results by the four evaluation indices are shown in Table 3 below. The confusion matrix of MdkResNet, which was high in all indices, is shown in Fig. 7. Table 3.3 shows the explanation of $\mathrm{A}$ to $\mathrm{L}$ in the legend of Fig. 7. The y-axis in Fig. 7 shows the correct labels, and the $\mathrm{x}$-axis shows the predicted labels by the trained model, making it easy to check the prediction results of the trained model.

\section{Conclusion}

In this study, we confirmed that the LSTM, the combination of the GAF algorithm and ResNet, and the combination of the GAF algorithm and Mdk-ResNet could be used to estimate the behavior of smartphones considering their possession position. As a result, we found that the combination of GAF algorithm and Mdk-ResNet has the highest accuracy.

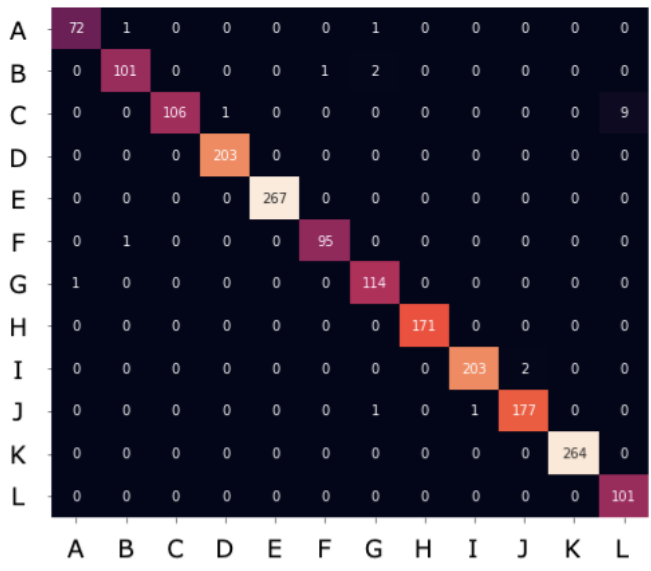

Figure 7: Confusion Matrix

In addition, compared to previous studies, it was confirmed that classification was possible with relatively high accuracy even when the possession position was taken into account. Also, because it is a classification system, even if there is input other than the set action or possession position, it will be classified into one of the 12 classes in this case. In the future, we plan to use deep metric learning and other methods to enable processing when there is input other than the predetermined action or possession position.

\section{References}

[1] S. Hochreiter and J. Schmidhuder, "Long Short-Term Memory", Neural Computation, Vol.9, No.8, pp.1735-1780, 1997. DOI: $10.1162 /$ neco.1997.9.8.1735

[2] Z. Wang and T. Oates, "Imaging Time-Series to Improve Classification and Imputation", In Proceedings of the 24th International Conference on Artificial Intelligence (IJCAI'15), pp.3939-3945, 2015. DOI: $10.5555 / 2832747.2832798$

[3] H. Kamining, X. Zhang, S. Ren and J. Sun, "Deep Residual Learning for Image Recognition", 2015. DOI: 10.1109/CVPR.2016.90 
[4] H. Xu et al., "Human Activity Recognition Based on Gramian Angular Field and Deep Convolutional Neural Network", in IEEE Access, Vol.8, pp.199393-199405, 2020. DOI: 10.1109/ACCESS.2020.3032699

[5] Release of "White Paper on Mobile Society 2021" $\sim$ Survey data summarizing trends in mobile ICT usage over the past 12 years is now available free of charge , NTTdocomo, https://www.nttdocomo.co.jp/binary/ pdf/info/news_release/topics_210914_00.pdf, access date: 2021.12 .13

[6] Consumer Confidence Survey, Department of Business Statistics Economic and Social Research Institute Cabinet Office, Government of Japan, https://www.esri.cao. go.jp/jp/stat/shouhi/honbun202103.pdf, access date: 2021.12 .13

[7] Investigation result summary, MOBILE MARKETING DATA LABO., https://mmdlabo.jp/investigation/ detail_1930.html, access date: 2021.12.13

[8] Smartwatch Sales in Japan Surpass 2 Million Units, MMRI, https://www.m2ri.jp/release/detail.html? id $=508$, access date: 2021.12 .13

[9] T. Iwamoto, D. Sugimori and M. Matsumoto, "A Study of Identification of Pedestrian by Using 3-axis Accelerometer", Information Processing Society of Japan, Transactions of the Information Processing Society of Japan, Vol.55, pp.739749,2014

[10] J. R. Kwapisz, G. M. Weiss, and S. A. Moore, "Activity recognition using cell phone accelerometers", ACM SIGKDD Explorations Newsletter, Vol.12, Issue.2 pp.74-82, 2010. DOI: $10.1145 / 1964897.1964918$

[11] F. Yu and V.Koltun, "Multi-Scale Context Aggregation by Dilated Convolutions", arXiv:1511.07122, 2015.

[12] C. Szegedy, W. Liu, Y. Jia, P. Sermanet, S. Reed, D. Anguelov, D. Erhan, V. Vanhoucke and A. Rabinovich, "Going Deeper with Convolutions", 2015 IEEE Conference on Computer Vision and Pattern Recognition (CVPR) pp.1-9, 2015, DOI: 10.1109/CVPR.2015.7298594

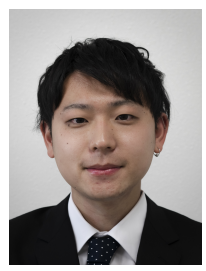

Rui Kitahara (Member) was born in Fukuoka, Japan, in 1996 and received B.S. degree in Electrical and Electronic Engineering from Kyushu Institute of Technology, Japan, in 2020. He is currently pursuing M.S. degree in Electrical and Electronic Engineering at Kyushu Institute of Technology, Japan. His current research interests include image classification using machine

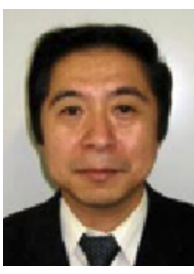

Seiichi Serikawa (Member) was born in $\mathrm{Ku}-$ mamoto Japan in June 1961. He received a B.S.degree and M.S. degree in electrical engineering from Kumamoto University in 1984 and 1986. He received a Ph.D. degree in electronic engineering from Kyushu Institute of Technology in 1994. Currently, he is a professor of the Department of electrical engineering and electronics at Kyushu Institute of Technology, Japan. His current research interests include computer vision, sensors, and robotics. He is a member of IEICE, IEEJ, IPSJ, IIAE.

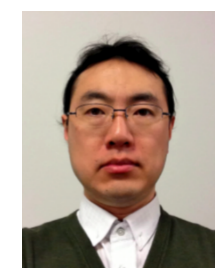

Lifeng Zhang (Member) received the B.S. degree in Electronic Engineering from Southeast University in 1994. He received M.S. and Ph.D. degrees in Electrical Engineering from Kyushu Institute of Technology, in 1999 and 2001, respectively. Now, he is a professor at Kyushu Institute of Technology. His current research interests include computer vision, image processing, biometrics authentication, and remote sensing system. He is a member of IIAE, IEEE, and IEICE.

learning. 